\title{
Editorial
}

\section{Microbiota in Human Health}

\section{Barua UK}

It has been long known that microorganisms in the human gut play an important role in digestive health. However, more recent research indicates that gut bacteria may relate to wider aspects of health, including obesity and metabolic health. Microorganisms inhabit various sites of the human body, in particular, the human gut where they are present in the form of: Eukarya, Bacteria, and Achaea as defined by their molecular phylogeny. Bacteria comprising $90 \%$, of these are more than 1,000 species and belong to Firmicutes and Bacteroidetes ${ }^{1,2}$. The gut microorganisms are approximately 100 trillion bacteria cells, outnumbering human cells by an estimated 10 fold $^{3}$. Each person has a distinct and highly variable composition of gut microbes, although a core set of microorganisms are common to all individuals ${ }^{1,4}$. The composition of the gut microorganisms is called the gut 'microbiota', whereas the totality of the genes of the microbiota is called the 'microbiome', which also outnumber the genes of the human body and is approximately 150 -folds ${ }^{3}$.

The foetus in the womb is sterile; it starts gaining microbiological organisms as time goes and begins even when it is passes through the birth canal and/or exposes to microbes present in the environment. Babies that are born by Caesarean have a different gut microbiota and increased risk of overweight and obesity in later life, compared to those delivered vaginally ${ }^{5}$. Although the microbiota is established in early life, it can shift throughout life with changes in age, diet, geographical location, intake of food supplements and drugs, and other environmental influences ${ }^{6}$. Excess body fat and disease are also associated with an altered gut microbiota.

Food habits shifting from breast feeding through formulafed to different composition adopted by people of varied ethnic groups in different geographic area have been shown to have impact on the composition of gut microbiota and thus on human health and diseases ${ }^{1}$.

Most research on the human microbiota focuses on the microorganisms in the gut, as they are thought to influence health in various ways. It has been documented that people, who suffer from certain diseases like inflammatory bowel disease, irritable bowel disease, allergy have a microbiota that is different to that of healthy people. The patterns of a gut microbiota that are associated with health are, however, more difficult to define ${ }^{6}$. The composition of the gut microbiota is highly variable even between healthy subjects. It has therefore been suggested that the function of the gut microbiota, rather than the composition, is more important for health ${ }^{6}$.

The microorganisms present in the gut play a crucial role in digestive health, but also influence the immune system. Immune tissues in the gastrointestinal tract constitute the largest and most complex fraction of the human immune system. The intestinal mucosa is a large surface lining the intestine and is exposed to pathogenic and non-pathogenic environmental antigens. In the gut lumen, the microorganisms play a critical role in the development of a robust and balanced immune system ${ }^{2}$. Alterations in an individual's gut microbiota, which can happen when taking certain antibiotics for example, can increase the risk of infections with opportunistic pathogens such as Clostridium difficile 6 .

In recent years researchers have established a link between the gut microbiota and body weight. Whether the altered microbiota composition is a cause or consequence of obesity is currently unknown. Studies show that the composition of the gut microbiota shifts with weight loss and/or weight gain ${ }^{4}$. But most of these evidences are from animal studies and so are debated and need further research, in particular in humans, to understand how and to what extent the composition of microorganisms in the gut influences various metabolic functions in the body.

Probiotics as defined are live microorganisms, which, when administered in adequate amounts, may confer a health benefit in certain conditions like Clostridium difficile associated diarrhea. Inflammatory Bowel Diseases and some allergic conditions like Asthma. But the evidence is still inconclusive and more studies will be needed to confirm benefits of probiotics. Prebiotics are non-

Correspondence: Prof. Dr. Uttam Kumar Barua, Professor \& Head Department of Respiratory Medicine, Shaheed Suhrawardy Medical College, SherE-Bangla Nagar, Dhaka-1207, Bangladesh; Email: dr_uk_barua@yahoo.com; Cell: +8801819402358 
digestible food components that are selectively used by gut bacteria for fermentation and thus are supposed to have health impact through augmenting or preventing the growth of a particular gut microorganism ${ }^{7}$.

The microorganisms present in the human gut are without doubt crucial for human health. Exactly how, to what extent, and what areas of human health are influenced by our "inhabitants" is yet to be established, as is the evidence on how the composition and/or function of the microbiota could be manipulated to achieve specific health benefits. [J Shaheed Suhrawardy Med Coll, 2014;6(1):1-2]

\section{References}

1. Tremaroli V, Backhed F. Functional interactions between the gut microbiota and host metabolism. Nature 2012; 489(7415): 242-249

2. Robles Alonso V, Guarner F. Linking the gut microbiota to human health. British Journal of Nutrition 2013; 109(S2): S21-S26
3. Wu GD, Lewis JD. Analysis of the Human Gut Microbiome and Association with Disease. Clinical Gastroenterology and Hepatology 2013; 11(7): 774-777

4. Molinaro F, Paschetta E, Cassader M, Gambino R, Musso G. Probiotics, prebiotics, energy balance, and obesity: mechanistic insights and therapeutic implications. Gastroenterology Clinics of North America 2012; 41(4): 843-854

5. Li HT, Zhou YB, Liu JM. The impact of cesarean section on offspring overweight and obesity: a systematic review and meta-analysis. International Journal of Obesity 2013; 37(7): 893-899

6. Backhed F, Fraser CM, Ringel Y, Sanders ME, Sartor RB, Sherman PM, et al. Defining a healthy human gut microbiome: current concepts, future directions, and clinical applications. Cell host \& microbe 2012;12(5):611-622

7. Hempel S, Newberry SJ, Maher AR, Wang Z, Miles JNV, Shanman R, et al. Probiotics for the prevention and treatment of antibiotic-associated diarrhea: a systematic review and meta-analysis. Jama 2012;307(18):1959-1969 